\title{
Thermally activated ferroelectric domain growth due to random defects
}

\author{
V. Likodimos, ${ }^{*}$ M. Labardi, X. K. Orlik, L. Pardi, and M. Allegrini ${ }^{\dagger}$ \\ INFM and Dipartimento di Fisica, Università di Pisa, Via F. Buonarroti 2, I-56127 Pisa, Italy \\ S. Emonin ${ }^{\ddagger}$ and O. Marti \\ Universität Ulm, Abteilung Experimentelle Physik, Albert-Einstein-Allee 11, D-89069 Ulm, Germany
}

(Received 12 July 2000; published 19 January 2001)

\begin{abstract}
Ferroelectric domain kinetics on cleaved triglycine sulfate, quenched at different temperatures in the ferroelectric phase, is investigated in situ by scanning force microscopy in the dynamic contact mode. Thermally activated domain growth and dynamic scaling, in accordance with theoretical predictions for quenched disorder due to random-bond defects, is inferred from the temporal evolution of the spatial correlation functions and the related characteristic length scale.
\end{abstract}

DOI: $10.1103 /$ PhysRevB.63.064104

PACS number(s): 77.80.Dj, 68.37.Ef, 64.60.My, 77.84.Fa

Defects are known to influence profoundly the domain structure and physical properties of ferroelectric crystals. ${ }^{1,2}$ Domain kinetics have been predicted to be severely hindered by randomly distributed defects and impurities that lead to slow logarithmic growth laws according to the type of quenched disorder, pertinent to the system's heterogeneity. ${ }^{3,4}$ Verification of these theoretical predictions has been experimentally challenging, ${ }^{5}$ seldom realized in disordered magnetic systems ${ }^{6-8}$ and in a nematic liquid crystal. ${ }^{9}$ Although the same predictions have been put forward for ferroelectrics, ${ }^{10}$ where power growth laws with exponents close to that of conserved systems have been rarely observed, ${ }^{11,12}$ experimental evidence for the validity of the nonalgebraic domain growth due to quenched disorder, to our knowledge, has not been reported.

Recent advances in scanning force microscopy (SFM) ${ }^{13}$ and other microscopic methods ${ }^{14,15}$ have offered unique opportunities for noninvasive, nanoscale imaging of domains on ferroelectric surfaces. In particular, voltage-modulated SFM, operated in the dynamic contact mode, providing sharp domain contrast well separated from topography, has been exploited for dynamical studies of ferroelectric domains. ${ }^{16-19}$ Very recently, its application to the study of domain kinetics on triglycine sulfate (TGS) cleavage surfaces, quenched from the paraelectric to the ferroelectric phase, has provided first evidence for a crossover to the logarithmic growth expected in the case of random-bond pinning forces and dynamic scaling in the ordering process. ${ }^{20}$

In this work, domain kinetics on cleaved TGS single crystals, quenched at different temperatures in the ferroelectric phase, is investigated. We show that thermally activated domain kinetics and dynamic scaling at the submicron scale, consistent with the logarithmic growth law predicted for systems with quenched random bonds, can be inferred directly from the time and temperature dependence of the characteristic correlation length.

Experiments were conducted in air using a home-built SFM employing the voltage-modulation technique in the dynamic contact mode. ${ }^{21}$ Domain detection relies on the application of an ac potential between the conductive probe tip and the rear sample electrode. Polarization-dependent forces of electric/electromechanical origin exerted on the probe tip, modulate the cantilever bending at the excitation frequency, detected by means of lock-in techniques. Conductive ( $\rho$ $\sim 0.01 \Omega \mathrm{cm}) \mathrm{n}^{+}$-silicon cantilevers appropriate for the contact operation mode (Nanosensors ${ }^{\mathrm{TM}}, 0.1 \mathrm{~N} / \mathrm{m}, 15 \mathrm{kHz}$ ) are used.

Domain contrast in dynamic contact SFM has been recently shown to depend markedly on the modulation frequency and exhibit pronounced resonance effects owing to the excitation of resonant modes of the cantilever in contact conditions. ${ }^{21}$ The applied voltage (peak-to-peak amplitude of $5 \mathrm{~V}$ ) is thus modulated at the resonance peak frequency of the contrast amplitude spectrum, in our case $64 \mathrm{kHz}$. This also ensures deconvolution of the enhanced polarization signal from topographic properties, which may inherently occur in other SFM modes. Measurements were performed on uniaxial TGS single crystals undergoing a typical secondorder phase transition to the ferroelectric phase at $T_{c}$ $\approx 322 \mathrm{~K},{ }^{1}$ where intrinsic defect centers have been found to influence substantially domain nucleation and the switching process of the spontaneous polarization. ${ }^{22}$ Slices (thickness $\approx 0.5-1 \mathrm{~mm}$ ) of a TGS crystal grown from aqueous solution at room temperature were cleaved along the (010) plane in air. In situ thermal treatment was performed using a temperature controlled stage $(\Delta T \leqslant 0.1 \mathrm{~K}),{ }^{17}$ while timedependent measurements were performed on a previously annealed specimen that exhibited relatively high domain density after prolonged exposure at ambient conditions.

Figure 1 summarizes the temporal evolution of domain patterns at the TGS (010) cleavage face for approximately $100 \mathrm{~min}$, after quenching at different temperatures $T<T_{c}$. Prior to each image sequence, the specimen was annealed at $333 \mathrm{~K}$ for $60 \mathrm{~min}$ and then rapidly cooled below $T_{c}$, within 1 to $2 \mathrm{~min}$. Once thermal equilibrium has been reached, the in-phase component of the ac cantilever response has been recorded in order to avoid the presence of different contrast at domain walls prevailing in the amplitude signal. ${ }^{21}$ The origin of time is set at the time of crossing $T_{c}$ upon cooling. Immediately after the quench, fine lamellar patterns of antiparallel ferroelectric domains, preferably oriented perpendicular to the $c$ axis, ${ }^{22}$ are detected with sharp contrast. The initial domain density exhibits a maximum at $313 \mathrm{~K}$. With the passage of time, domain images reveal coarser, though 


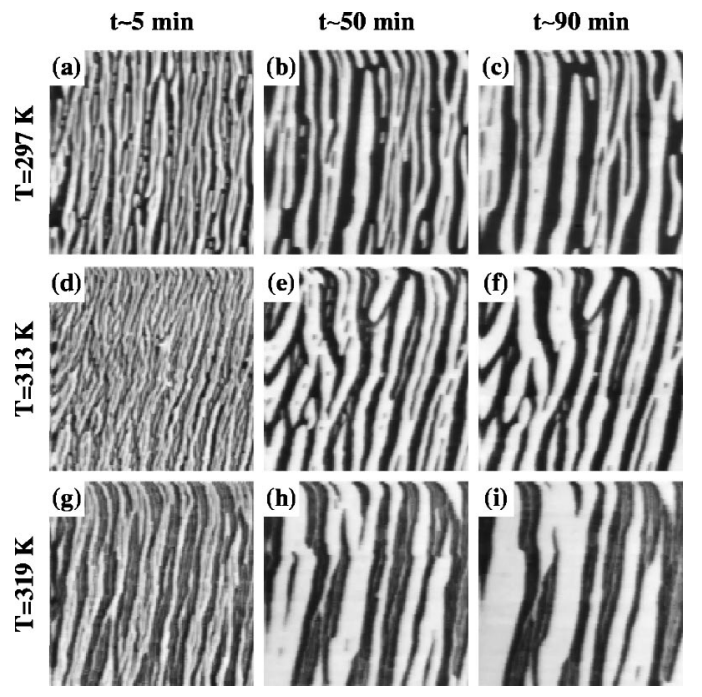

FIG. 1. Domain images for the (010) TGS surface following a rapid quench at different temperatures in the ferroelectric phase. Images have been recorded acquiring the in-phase component of the cantilever response: (a) $6 \mathrm{~min}$, (b) $50 \mathrm{~min}$, (c) $97 \mathrm{~min}$, (d) $4 \mathrm{~min}$, (e) $54 \mathrm{~min}$, (f) $92 \mathrm{~min}$, (g) $9 \mathrm{~min}$, (h) $51 \mathrm{~min}$, and (i) $90 \mathrm{~min}$, after crossing $T_{c}$. Scan size $20 \times 20 \mu \mathrm{m}^{2}$; modulation frequency 64 $\mathrm{kHz}$.

morphologically self-similar, patterns. The underlying evolution proceeds by the sideways wall motion that leads to shrinkage of the smaller nuclei and coalescence of adjacent stripes, implying a curvature-driven growth mechanism due to surface tension. ${ }^{23}$

To quantify growth kinetics, we have calculated the spatially averaged equal-time correlation function $C(r, t)$ $\equiv\langle S(r, t) S(0, t)\rangle$, where $S(r, t)$ corresponds to the scalar order parameter field, ${ }^{23}$ in accordance with the Ising-type definition formally used to describe order-disorder phase transitions as for uniaxial TGS, ${ }^{2}$ and assumes either of the values +1 and -1 for $r$ residing in each of the two domain states. The correlation functions were computed along the horizontal image direction, about perpendicular to the domain walls, as a function of $r$, for all times and temperatures. For short distances, $C(r, t)$ is found to decay rapidly as a function of $r$, at a rate that becomes much slower with the passage of time, implying nonlinear coarsening processes. For longer distances, oscillations appear on the tail of $C(r, t)$ reflecting the periodic structure of the lamellar pattern along the $c$ axis, consistent with the anisotropy of the TGS wall energy ${ }^{22}$ and previous reports. ${ }^{11,17,20}$ The time dependence of the average domain size was derived from the temporal evolution of the characteristic length scale $L(t)$ defined as the distance where $C(r, t)$ drops to half of its value at $r=0$, i.e., $C[r=L(t), t]$ $=1 / 2$, for a given time $t$. The corresponding correlation length $L(t)$ has been previously used to deduce the average domain size on TGS domain patterns, yielding equivalent growth laws parallel and perpendicular to the $c$ axis on submillimeter scales, ${ }^{11}$ while, recently, it has been found to scale consistently as the inverse of the total domain wall length for TGS domain patterns at the submicron range, ${ }^{20}$ and thus provide a reliable probe of the average domain width in real time. The resulting growth law at different temperatures is

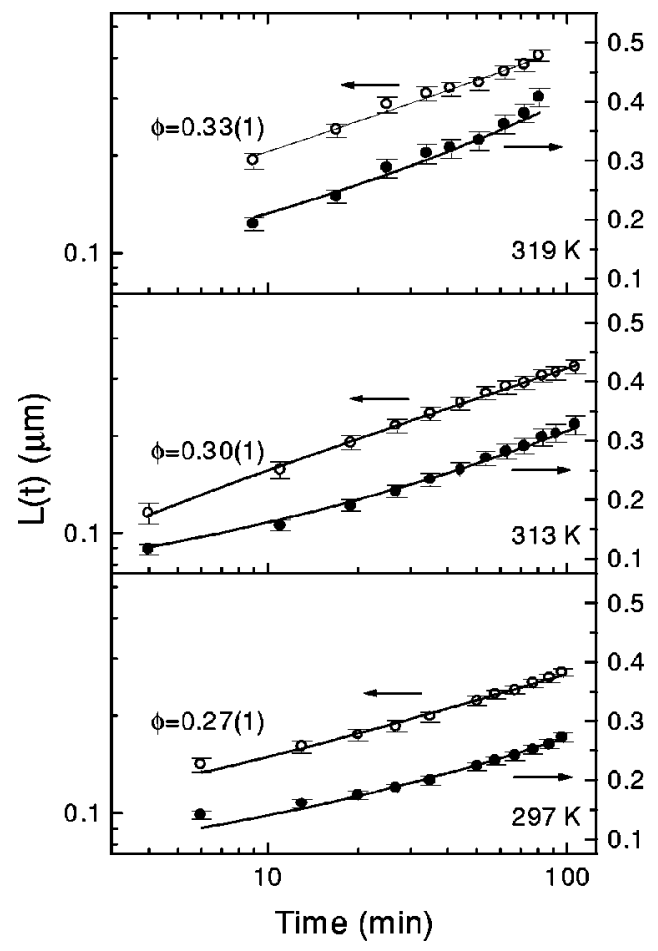

FIG. 2. Time dependence of the correlation length $L(t)$ at different temperatures below $T_{c}$ in logarithmic (open circles) and semilogarithmic (solid circles) scales. Solid lines correspond to the best-fit curves to the power-law dependence $L(t) \propto\left(t-t_{0}\right)^{\phi}$ with $t_{0}=0(1) \mathrm{min}$ and exponent $\phi$ values indicated for each temperature. Dashed lines correspond to the best fit curves to the logarithmic growth law $L(t) \sim A(T)\left[\ln \left(t / \tau_{0}\right)\right]^{4}$ with $\tau_{0}=18 \times 10^{-4} \mathrm{~s}$ and the amplitude values reported in the text.

plotted in logarithmic and semilogarithmic scales in Fig. 2. Errors in the $L(t)$ measurement were calculated on account of effects due to filtering, threshold level choice, and temporal drift of the imaging frame.

The temporal variation of $L(t)$ can be described with a power-law dependence $L(t) \propto\left(t-t_{0}\right)^{\phi}$, with time offset values $t_{0}=0(1) \mathrm{min}$. The growth exponents inferred from the $\log$-log plots are close to $1 / 3$, in agreement with previous results derived by different experimental methods for triglycine sulfate on both the submillimeter ${ }^{11}$ and recently submicron scales, ${ }^{20}$ as well as the strongly anisotropic domain patterns of sodium nitrite. ${ }^{12}$ Further, the domain area ratio for each polarization state is preserved to within $6 \%$ of a mean value of 0.5 , in the present time and spatial scale. These exponent values along with the conservation of the domain area appear to comply with the presence of conserved dynamics, ${ }^{11,20}$ which, however, may be regarded as fortuitous with respect to implications of the long-range electrostatic interaction on polarization that is formally a nonconserved order parameter. ${ }^{12}$ On the other hand, the most remarkable feature of the observed $L(t)$ variation is a small, though substantial, temperature dependence of the growth exponents, implying faster dynamics as temperature increases. The origin of this behavior can be described within the activated scaling dynamics predicted for quenched random media, where domain growth proceeds through the in- 
terplay between the driving force due to surface tension and pinning forces induced by random defects. ${ }^{3-5,10}$

Thorough theoretical studies have established that domain relaxation due to quenched disorder is dominated by thermal activation over energy barriers leading to slow logarithmic domain growth. In this case, the typical transverse displacement of a segment of a wall of length $l$, is of the order $l^{\zeta}$, where $\zeta$ is the roughness exponent $(0 \leqslant \zeta<1) .{ }^{3,4}$ The wall energy, estimated as elastic energy of the deformed wall, is then predicted to be of the order $l^{\chi}$ with $\chi=d-3+2 \zeta$, where $d$ is the spatial dimensionality, provided that pinning and elastic energies are comparable. Assuming that domain walls with a typical radius of curvature $L$ move in linear sections of length $l$ and that their distortion due to curvature, which is of the order $l^{2} / L$, is comparable with the disorder roughening $l^{\zeta}$, the two lengths relate as $l \sim L^{1 /(2-\zeta)}$. The same scaling relation as well as the lower bounds imposed on $l$ by the intrinsic wall width and the defect size can be also deduced considering the interplay between the pinning force and the Laplace pressure due to surface tension. ${ }^{5,10}$ The barriers to domain motion are then found to scale as $E(L)$ $\sim L^{\chi /(2-\zeta)}$. According to the Arrhenius law, the time required to surmount the energy barriers is $t(L)$ $\sim \tau_{0} \exp [E(L) / k T]$, where $\tau_{0}$ is a microscopic time, leading to the logarithmic time dependence of the average domain size $L(t) \sim\left[T \ln \left(t / \tau_{0}\right)\right]^{(2-\zeta) / \chi}$ (Ref. 4). The latter can be directly compared with the characteristic length scale $L(t)$ measuring the average domain width of the lamellar TGS domain patterns as imposed by the anisotropic wall energy (Fig. 2).

For defects of the random-field type, directly coupled to the order parameter, and $d=2$ applying to a "thin" ferroelectric plate on account of the long-range Coulomb fields, ${ }^{10}$ the roughness exponent has been predicted to be $\zeta=1$ (Ref. 3 ). On the other hand, for random-bond type defects, which couple to the local energy, regardless of the domain state, the exponent value of $\zeta=2 / 3$ has been determined. ${ }^{4,24}$ Fitting of the experimental $L(t)$ data to the corresponding growth laws, namely $L(t) \sim\left[T \ln \left(t / \tau_{0}\right)\right]$ for the random-field model and $L(t) \sim\left[T \ln \left(t / \tau_{0}\right)\right]^{4}$ for the random-bond one, yields reasonably good fit only in the latter case, as can be inferred from the nonlinear $L(t)$ dependence in the semilogarithmic scales of Fig. 2. Accordingly, the $L(t)$ dependence has been fitted to the expression $L(t) \sim A(T)\left[\ln \left(t / \tau_{0}\right)\right]^{4}$, where the amplitude $A(T)$ should scale as $T^{4}$, were the random-bond model predictions to be fully recovered. Keeping a constant value for the microscopic time $\tau_{0}=18 \times 10^{-4} \mathrm{~s}$, a fit of the three data sets yields $A(T)$ values of 15,18 , and $20 \times 10^{-4} \mu \mathrm{m}$ at 297, 313, and $319 \mathrm{~K}$, respectively. Comparison of all possible ratios of the $A(T)$ amplitude values, $A(319) / A(297)$ $=1.33, A(319) / A(313)=1.11$, and $A(313) / A(297)=1.20$, with the corresponding temperature ratios in the fourth power, namely, $1.33,1.08$, and 1.23 , shows that the experimental $L(t)$ dependence is indeed in good agreement with the specific temperature variation that inherently appears in the growth law for quenched random bonds.

An important theoretical prediction for domain growth in the presence of quenched disorder concerns the development of a scaling regime at long times after the quench. ${ }^{23,25,26}$ This dynamic scaling turns out to be independent of pinning

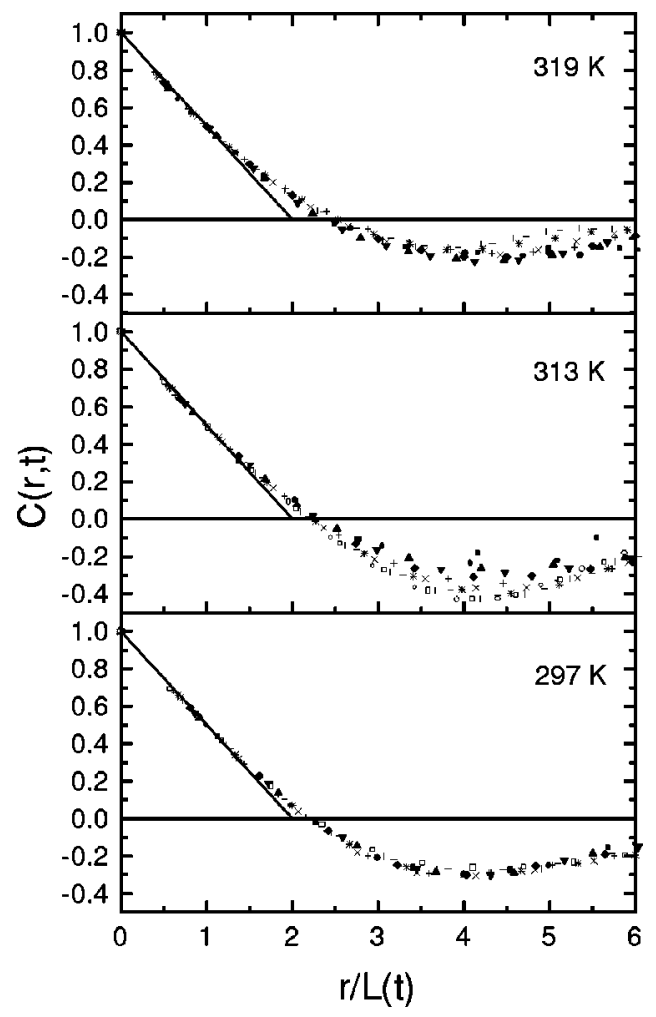

FIG. 3. Scaled correlation functions $C(r, t)=f[x \equiv r / L(t)]$ of the TGS domain patterns at different temperatures. Solid symbols correspond to domain images taken at different times. Solid lines correspond to the linearly decreasing trend of the short-distance part of the scaling function according to $f(x)=1-0.5 x$.

forces that merely cause a renormalization of the bare kinetic coefficient at short-length scales. Accordingly, the equaltime correlation function has been predicted to obey the scaling form $C(r, t)=f[x \equiv r / L(t)]$, with the scaling function $f(x)$ independent of the strength of randomness and consistent with that of the pure systems. Figure 3 shows plots of the correlation functions $C(r, t)$ as a function of the scaled distance $x$ for all the different times and temperatures. All plots collapse onto a single curve up to a scaled distance of about 2, indicative of dynamic scaling. Apart from a slight sublinear curvature, the short-distance part of the scaling function decreases almost linearly with $x$ according to $f(x)$ $=1-0.5 x$ (Fig. 3). The latter behavior, implying a powerlaw tail in the dynamic structure factor for large wave vectors known as Porod's law, ${ }^{23}$ is in close agreement with theoretical predictions and numerical simulations for the linear coefficient of $f(x)$ in the two-dimensional Ising $\operatorname{model}^{27}$ and the scaling function experimentally derived from the SFM study of quenched TGS. ${ }^{20}$

Nevertheless, a complicated behavior of both scaling functions and growth law, implying different length scales in the growth process, has been encountered at longer times. However, it could not be reliably assessed due to finite-size effects in the imaging frame that distort the correlation functions in an unpredicted manner. To this extent, we may conclude that thermally activated domain growth over scaledependent barriers due to random-bond pinning forces can 
be consistently realized in the spatial and time regime studied for the cleavage surface of a TGS ferroelectric crystal.

In conclusion, direct experimental evidence for thermally activated domain kinetics due to random defects is revealed on cleaved TGS crystals, quenched in the ferroelectric phase, applying dynamic contact SFM. The time dependence of the characteristic length compares favorably with the logarith- mic growth law predicted for random-bond quenched disorder and its amplitude scales consistently with the underlying temperature dependence, while dynamic scaling is found in the studied time scale.

We gratefully acknowledge N. Garcia (CSIC-Madrid) for kindly providing the TGS samples and EC for financial support within the TMR Network ERBFMRXCT98-0242.
*Present address: Institute of Material Science, NCSR “Demokritos,' 15310 Aghia Paraskevi, Athens, Greece.

†On sabbatical leave from Dipartimento di Fisica della Materia e TFA, Universitá di Messina, Salita Sperone 31, I-98166 Messina, Italy.

†Present address: Université de Technologie de Troyes, Laboratoire de Nanotechnologie et d' Instrumentation Optique, rue Marie $\mathrm{Cu}-$ rie 12, B.P. 2060, 10010 Troyes Cedex, France.

${ }^{1}$ M. E. Lines and A. M. Glass, Principles and Applications of Ferroelectrics and Related Materials (Clarendon, Oxford, 1977).

${ }^{2}$ B. A. Strukov and A. P. Levanyuk, Ferroelectric Phenomena in Crystals (Springer, Berlin, 1998).

${ }^{3}$ J. Villain, Phys. Rev. Lett. 52, 1543 (1984).

${ }^{4}$ D. A. Huse and C. L. Henley, Phys. Rev. Lett. 54, 2708 (1985).

${ }^{5}$ T. Nattermann and I. Vilfan, Phys. Rev. Lett. 61, 223 (1988).

${ }^{6}$ H. Ikeda, Y. Endoh, and S. Itoh, Phys. Rev. Lett. 64, 1266 (1990).

${ }^{7}$ G. Schins, A. F. M. Arts, and H. W. de Wijn, Phys. Rev. Lett. 70, 2340 (1993).

${ }^{8}$ M. Lederman, J. V. Selinger, R. Bruinsma, R. Orbach, and J. Hammann, Phys. Rev. B 48, 3810 (1993).

${ }^{9}$ D. K. Shenoy, J. V. Selinger, K. A. Grüneberg, J. Naciri, and R. Shashidhar, Phys. Rev. Lett. 82, 1716 (1999).

${ }^{10}$ E. B. Kolomeisky, A. P. Levanyuk, and A. S. Sigov, Ferroelectrics 104, 195 (1990).

${ }^{11}$ N. Tomita, H. Orihara, and Y. Ishibashi, J. Phys. Soc. Jpn. 58, 1190 (1989); H. Orihara, N. Tomita, and Y. Ishibashi, Ferroelectrics 95, 45 (1989), and references therein.
${ }^{12}$ K. Hamano, J. Zhang, K. Abe, T. Mitsui, H. Sakata, and K. Ema, J. Phys. Soc. Jpn. 65, 142 (1996); J. Zhang, K. Hamano, K. Abe, T. Mitsui, H. Sakata, and K. Ema, ibid. 65, 149 (1996).

${ }^{13}$ F. Saurenbach and B. D. Terris, Appl. Phys. Lett. 56, 1703 (1990); R. Lüthi, H. Haefke, K.-P. Meyer, E. Meyer, L. Howald, and H. -J. Güntherodt, J. Appl. Phys. 74, 7461 (1993).

${ }^{14}$ S. Zhu and W. Cao, Phys. Rev. Lett. 79, 2558 (1997).

${ }^{15}$ T. J. Yang, V. Gopalan, P. J. Swart, and U. Mohideen, Phys. Rev. Lett. 82, 4106 (1999).

${ }^{16}$ J. W. Hong, K. H. Noh, S. -I. Park, S. I. Kwun, and Z. G. Khim, Phys. Rev. B 58, 5078 (1998).

${ }^{17}$ V. Likodimos, X. K. Orlik, M. Labardi, L. Pardi, and M. Allegrini, J. Appl. Phys. 87, 443 (2000).

${ }^{18}$ X. K. Orlik, V. Likodimos, M. Labardi, L. Pardi, and M. Allegrini, Appl. Phys. Lett. 76, 1321 (2000).

${ }^{19}$ E. Z. Luo, Z. Xie, J. B. Xu, I. H. Wilson, and L. H. Zhao, Phys. Rev. B 61, 203 (2000).

${ }^{20}$ V. Likodimos, M. Labardi, and M. Allegrini, Phys. Rev. B 61, 14 440 (2000).

${ }^{21}$ M. Labardi, V. Likodimos, and M. Allegrini, Phys. Rev. B 61, 14 390 (2000).

${ }^{22}$ L. I. Dontsova, N. A. Tikhomirova, and L. A. Shuvalov, Kristallografiya 39, 158 (1994) [Crystallogr. Rep. 39, 140 (1994)].

${ }^{23}$ A. J. Bray, Adv. Phys. 43, 357 (1994), and references therein.

${ }^{24}$ D. A. Huse, C. L. Henley, and D. S. Fisher, Phys. Rev. Lett. 55, 2924 (1985).

${ }^{25}$ M. Rao and A. Chakrabarti, Phys. Rev. Lett. 71, 3501 (1993).

${ }^{26}$ H. Hayakawa, Phys. Rev. B 47, 11696 (1993).

${ }^{27}$ A. J. Bray and K. Humayun, Phys. Rev. E 47, R9 (1993). 